\title{
Blocked shape memory effect in negative Poisson's ratio polymer metamaterials
}

\author{
Katarzyna Boba ${ }^{1}$, Matteo Bianchi ${ }^{1}$, Greg McCombe ${ }^{1}$, Ruben Gatt ${ }^{2}$, Anselm C. \\ Griffin $^{3}$, Robert M. Richardson ${ }^{4}$, Fabrizio Scarpa ${ }^{1}$,†, lan Hamerton ${ }^{1}$, \\ Joseph N. Grima², \\ ${ }^{1}$ Advanced Composites Centre for Innovation and Science (ACCIS), University of Bristol, BS8 \\ ITR Bristol UK, ${ }^{2}$ Metamaterials Unit, University of Malta, Msida MSD 2080, Malta, ${ }^{3}$ Materials \\ Science and Engineering, Georgia Institute of Technology, 801 Ferst Drive, N. W., Atlanta, GA \\ 30332-029, and ${ }^{4}$ H H Wills Physics Laboratory, University of Bristol, Tyndall Avenue, Bristol, \\ BS8 1TS, UK \\ E-mail: f.scarpa@bristol.ac.uk
}

\begin{abstract}
We describe a new class of negative Poisson's ratio (NPR) open cell PU-PE foams produced by blocking the shape memory effect in the polymer. Contrary to classical NPR open cell thermoset and thermoplastic foams that return to their auxetic phase after reheating (and therefore limit their use in technological applications), this new class of cellular solids has a permanent negative Poisson's ratio behavior, generated through multiple shape memory (mSM) treatments that lead to a fixity of the topology of the cell foam. The mSMNPR foams have Poisson's ratio values similar to the auxetic foams prior their return to the conventional phase, but compressive stress-strain curves similar to the ones of conventional foams. The results show that by manipulating the shape memory effect in polymer microstructures it is possible to obtain new classes of materials with unusual deformation mechanisms.
\end{abstract}


Keywords: shape memory, negative Poisson's ratio, polyurethane, foam, metamaterial

$\dagger$ To whom correspondence should be addressed 


\section{Introduction}

Shape memory (SM) is an attribute of materials that return to their original shape after a plastic deformation. SM is achieved through exposure to various stimuli, typically a heat source. Research on properties and application of SM materials is very extensive and includes shape memory alloys (SMA) $)^{1-4}$, recently emerging shape memory hybrids $(\mathrm{SMHs})^{5-8}$, and polymers (SMP) ${ }^{9}$. SMPs feature many advantages compared to SMAs, such as low density (usually from 1.0 to $1.3 \mathrm{~g} \mathrm{~cm}^{-3}$ ), higher strain recovery (maximum return rate over $400 \%$ ), and relatively low manufacturing costs with easy processing 5 . The polymers can be modified using relatively straightforward processes, allowing an easy adjustment of the polymers' properties according to requirements of the specific applications. The most commonly used SMP materials are thermoplastic polyurethanes (PU), due to its ease of preparation, high resistance to organic solvents and aqueous solutions, possible biocompatibility and biodegradability. Additionally, PU glass transition temperature can be easily tailored for the desired application. Foams constitute a specific subset of SMPs. Shape Memory Foams (SMFs) are manufactured in a similar to conventional foams how- ever, using SMP as their base materials. The first shape memory foam was proposed by Tey et al. ${ }^{10}$, using SMP core material in a open cell structure. Typical constituent solids for SMFs are epoxy ${ }^{11,12}$, polyurethane ${ }^{13}$ and vinyl copolymer-based polymers ${ }^{14}$. SMF have a very low density, are highly compressible and possess a significant shape recovery ratio that is very desirable for various applications, specifically in aerospace and automotive industries ${ }^{12,15}$. With SMFs, polymeric vehicles or structures could in theory be compacted during transportation and returned back to their original shape when delivered, significantly reducing the storage required for transportation ${ }^{11}$. 
Negative Poisson's ratio foams (NPR) polyurethane foams were first manufactured by Lakes using a combination of multiaxial compression of pristine foam, followed by heating below the polymer softening temperature and successive cooling ${ }^{16}$. The resultant foam features pores with a general re-entrant structure, providing the negative Poisson's ratio effect. Research in negative Poisson's ratio materials is not only stimulated by the counter-intuitive behavior of such solids, but also because of the large number of beneficial properties which may be used in practical applications, like increased indentation resistance, shear stiffness, plane strain fracture toughness, enhanced acoustic properties and the ability to form synclastic curvature. Negative Poisson's ratio foams may therefore perform better than their conventional counterparts in a number of products, including cushioning applications where the ability of the foam to form dome shaped structures will ease problems, such as pressure ulcers endured by persons who are confined on a wheel chair or are bed ridden. Negative Poisson's ratio foams may also show superior qualities when used in protection equipment because their material tends to flow towards the impact zone (i.e., it becomes denser). Porous filters may also benefit from the introduction of a negative Poisson's ratio since the area of the pores in auxetic materials - opposite to conventional solids tend to increase on the application of a stress. Since the publication of Lakes' seminal paper, different types of manufacturing processes have been designed to produce NPR open cell foams ${ }^{17-20}$. A shape memory effect can be also induced in negative Poisson's ratio foam specimens by applying a specific temperature profile, causing the dimensions of the NPR foam to return to their original values (conversion to returned phase). The process of converting PU foam from conventional to NPR (also denominated as auxetic ${ }^{21}$ ), and the subsequent reconversion to the returned (or original) phase, is designated as one conversion cycle ${ }^{22}$. Bianchi et $a l{ }^{22}$ have investigated the cyclic compressive and tensile properties of open cell PU foam at each stage of two successive conversion cycles, obtaining four different phases (native, $1^{\text {st }}$ auxetic, $1^{\text {st }}$ returned, $2^{\text {nd }}$ auxetic). A chemical and structural characterization of the PU base foam and its auxetic and $1^{\text {st }}$ returned phases has been recently performed by Li and Zeng ${ }^{23}$. In Bianchi et al. work the PU foam was found to possess similar Poisson's ratio values between the native and the $1^{\text {st }}$ returned phase, as well as between the $1^{\text {st }}$ and $2^{\text {nd }}$ auxetic phases. Significant differences were however observed about the energy dissipated per unit volume under cyclic loading and the tan- 
gent modulus of the foams, leaving open the question whether further SM treatments could provide other peculiar behaviors in the mechanical properties of the foams. Although NPR foams are envisaged to show all the aforementioned mechanical and multifunctional advantages, they suffer an important drawback due to their shape memory effect, due to which they revert back to their original structure (having a positive Poisson's ratio) when exposed to heat or solvents.

We describe in this paper how upon repeated shape memory cycles it is possible to transform a conventional polyurethane foam material permanently into a negative Poisson's ratio one even after nominal shape memory recovery. The irreversible (or blocked) shape memory effect generated in these open cell polyurethane foams can be used to produce designer polymeric porous materials with unusual deformation mechanisms (metamaterials) for applications ranging from higher temperature acoustic liners to biomedical implants in which negative Poisson's ratio porous polymers with high indentation and wear and tear properties. 


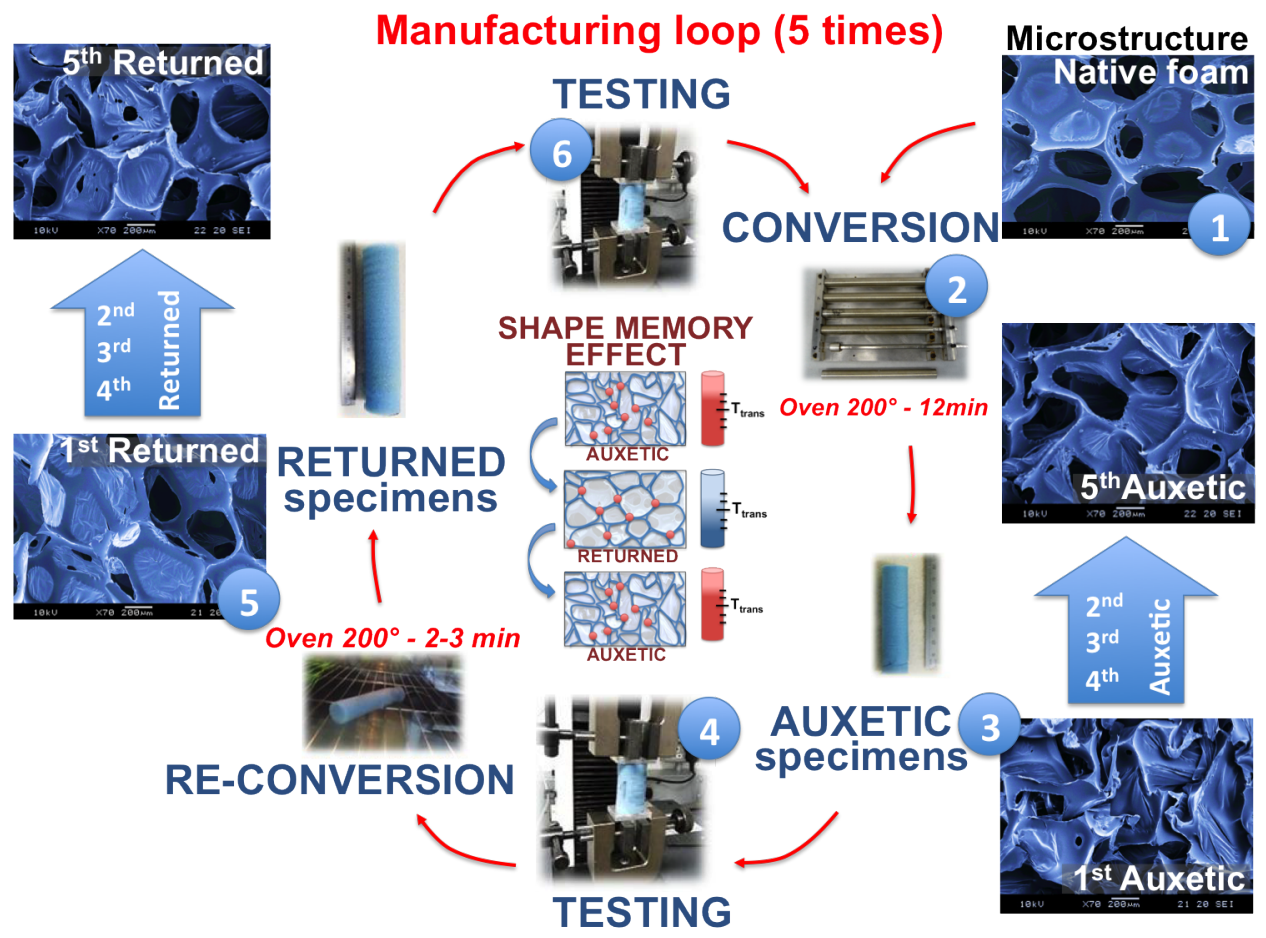

Figure 1: The multiple shape memory effect of auxetic foams. Conventional PU-PE open cell foam (1) is subjected to mechanical volumetric compression and annealing (2), obtaining a foam with negative Poisson's ratio characteristics (3). After being subjected to mechanical testing (4), the foam is placed in a oven at a temperature above the softening point, and returns to a conventional phase (5) with further testing (6). The returned-conventional foam is subjected to mechanical testing again. The conventional-auxetic-returned processing is performed five times. We obtain five auxetic phases (and five returned foam batches $\left(1^{\text {st }}, 2^{\text {nd }}, 3^{\text {rd }}, 4^{\text {th }}, 5^{\text {th }}\right)$. 


\section{Manufacturing and testing}

We have used five identical off-the-shelf open-cell PU-based conventional foam specimen types with $27 \mathrm{~kg} \mathrm{~m}^{-3}$ density (SM Upholstery Ltd, Cardiff, UK). The specimens had initial dimensions of $30 \mathrm{~mm}$ diameter and $160 \mathrm{~mm}$ in length (Figure 1). The pristine foams have been converted into the NPR (or auxetic) phase using the classical manufacturing route described $i^{24,25}$. The specimens have been compressed to their $50 \%$ and $37 \%$ of the original dimensions along the axial (i.e. foam length) and the radial (i.e. foam diameter) directions, respectively. The mould has been then placed into a preheated industrial oven Carbolite GP $450 \mathrm{~B}$ at $200^{\circ} \mathrm{C}$, according to the thermal $\beta$ profile described $i^{24}$. The temperature inside the foam specimen has been monitored using a thermocouple. Once the final temperature of $150^{\circ} \mathrm{C}$ after 12 mins has been reached, the mould was retrieved from the oven and cooled down at the room temperature for two hours. At the completion of the cooling process, the auxetic specimens have been extracted from the mould and gently pulled along their longitudinal direction to relax the samples from any residual stress on the outer surface ${ }^{24}$. The ends of the foams extracted from the mould have also been cut before testing, to obtain specimen with homogeneous mechanical and structural properties. NPR foams show a natural tendency to partially recover their original dimensions ${ }^{24}$, therefore all the density measurements were performed immediately after manufacturing. Following 22 the auxetic foam specimens were placed into a preheated oven at $200^{\circ} \mathrm{C}$. For all the samples we have observed a re- laxation process starting to be visible when the foam specimen core reached $100^{\circ} \mathrm{C}$. The relaxation rate rapidly increases until a temperature of $150^{\circ} \mathrm{C}$ was reached, and full recovery of the original dimensions is obtained. The specimens in this first new phase are named as "returned"17. The cyclic process of conversion into auxetic and back into returned phase has been repeated five times, with 11 different states for the foams considered. Five samples have been converted within each production batch. The mechanical tests have bee carried out immediately after the manufacturing process. The dimensions of the foams have been measured using a digital caliper (sensitivity $0.01 \mathrm{~mm}$ ) at different location of the sample length.

The specimens have been also weighted using an electronic scale (sensitivity of $0.001 \mathrm{~g}$ ). The 
final density ratio of the foams has been calculated as ${ }^{26} F D R=\rho / \rho_{\text {CONV }}$, where $\rho_{\text {conv }}$ is the density of the pristine foam $\left(27 \mathrm{kgm}^{-3}\right)$ and $\rho$ is the actual value of foam density at each different phase. Cyclic compressive loading has been performed at a displacement rate of 20 $\mathrm{mmmin}^{-1}$ using a Instron 3343 testing machine equipped with $1 \mathrm{kN}$ load cell. Each test consisted in five cycles at $10 \%$ of maximum compressive strain. During testing, the longitudinal and radial displacements have been measured using an Imetrum Video Gauge System with highresolution monochrome camera $(0.5 \mu \mathrm{m}$ over $10 \mathrm{~mm}$ of maximum displacement). The cameras was equipped with telecentric lens and supported by a low energy fluorescent lamp giving a cool and diffuse light. Tracking targets for the measurement of the displacement via the video gauge have been placed in three different positions around the centre of the sample (i.e. the surface of the foams free from end effects). The surfaces of the samples have been also marked in black to increase the contrast and facilitate the data acquisition. Measurements were taken only from the $5^{\text {th }}$ cycle to avoid effects from possible failed ribs during the manufacturing ${ }^{18-}$ 20,27,28. The Poisson's ratio was calculated from the longitudinal and radial strains at the $10 \%$ value of compressive strain following the classical definition $v_{z r}=-\varepsilon_{r} / \varepsilon_{z}{ }^{26}$. The tangent modulus $\left(E_{T}\right)$ has been calculated from the linear region of the stress-strain curve of the foams (both conventional and auxetic) at $10 \%$ of compressive $\operatorname{strain}^{26}$ as $E_{T}=d \sigma / d \varepsilon$.

Values of the energy dissipated through hysteresis within a cyclic loading were calculated approximating the sides of each stress-strain curve with two second degree polynomial curve followed by numerical integration of areas. Differential Scanning Calorimetry (DSC) has been also carried out using a TA Instruments Q100 DS Calorimeter. The DSC analysis has been performed on all the foams from the $1^{\text {st }}$ to the $5^{\text {th }}$ returned cycle, as well as on the native (pristine) foam. An elemental analysis $(\mathrm{C} / \mathrm{H} / \mathrm{N})$ has been also carried out on the foam samples. The microstructure of the foams has been observed using a Scanning Electron Microscope (JEOL JSM-5600LV) at $10 \mathrm{kV}$ of accelerating voltage. X-Ray Computed Tomography (CT) scanning was performed on a sample belonging to the $5^{\text {th }}$ returned phase and the pristine foam, using Nikon-Xtek $320 \mathrm{kV}$ machine. Specimens were scanned at $54 \mathrm{kV}$ and $249 \mathrm{~mA}$ with a resolution of $18 \mu \mathrm{m}$. IR spectroscopy of the foam specimens has been carried out using a 
Elmer Perkin Spectrum 100 FTIR facility at 400 spectral lines. The SAXS and WAXS tests were performed using a Ganesha SAXS instrument (http://saxsolutions.e-monsite.com/). The samples were contained in flat plate cells with mica windows and O-ring seals. The $Q$ range covered was within $0.003 \AA$ and $3 \AA(200 \mathrm{~nm}$ to 2 $\mathrm{nm})$. The tests were carried out at room temperature $\left(23^{\circ} \mathrm{C}\right)$. The data coming from SAXS and WAXS measurements were postprocessed using the Lorentz polarization correction $1 / \mathrm{Q}^{2} \mathrm{P}$, with $\mathrm{P}$ $=0.5+0.5 \cos ^{2}(2 \theta)$.

\section{Results and discussions}

The microstructure of the foam in the auxetic states has a typical re-entrant shape, and is clearly different from the one belonging to the pristine phase (Figure 1). Significant changes from the microstructure of the pristine foam are however observed for the returned phases. The foam in the initial condition shows a reticulated topology with rounded cells and the presence of membranes, while the cells in the returned specimens appear to be deformed from this more smooth and regular microstructure, with creases in the membranes and kinks along their ribs, thereby confirming previous reports ${ }^{22}$. From the $3^{\text {rd }}$ phase onwards the microstructure of the returned foams is however even more convoluted, and ribs with corrugated/non-uniform cross section are also present. The diameter of the specimens also changes throughout the SM process, with the nominal dimensions varying from $94 \%$ of the original diameter for the $1^{\text {st }}$ returned foam to $85 \%$ for the last. According to a two-tailed student's T-test, these values are statistically significant. No changes in sample dimensions are observed for the specimens in the NPR form. This is expected, given the fixed mould dimensions used in this work. Statistically relevant density changes ${ }^{17}$ reaching a maximum of $55 \%$ 

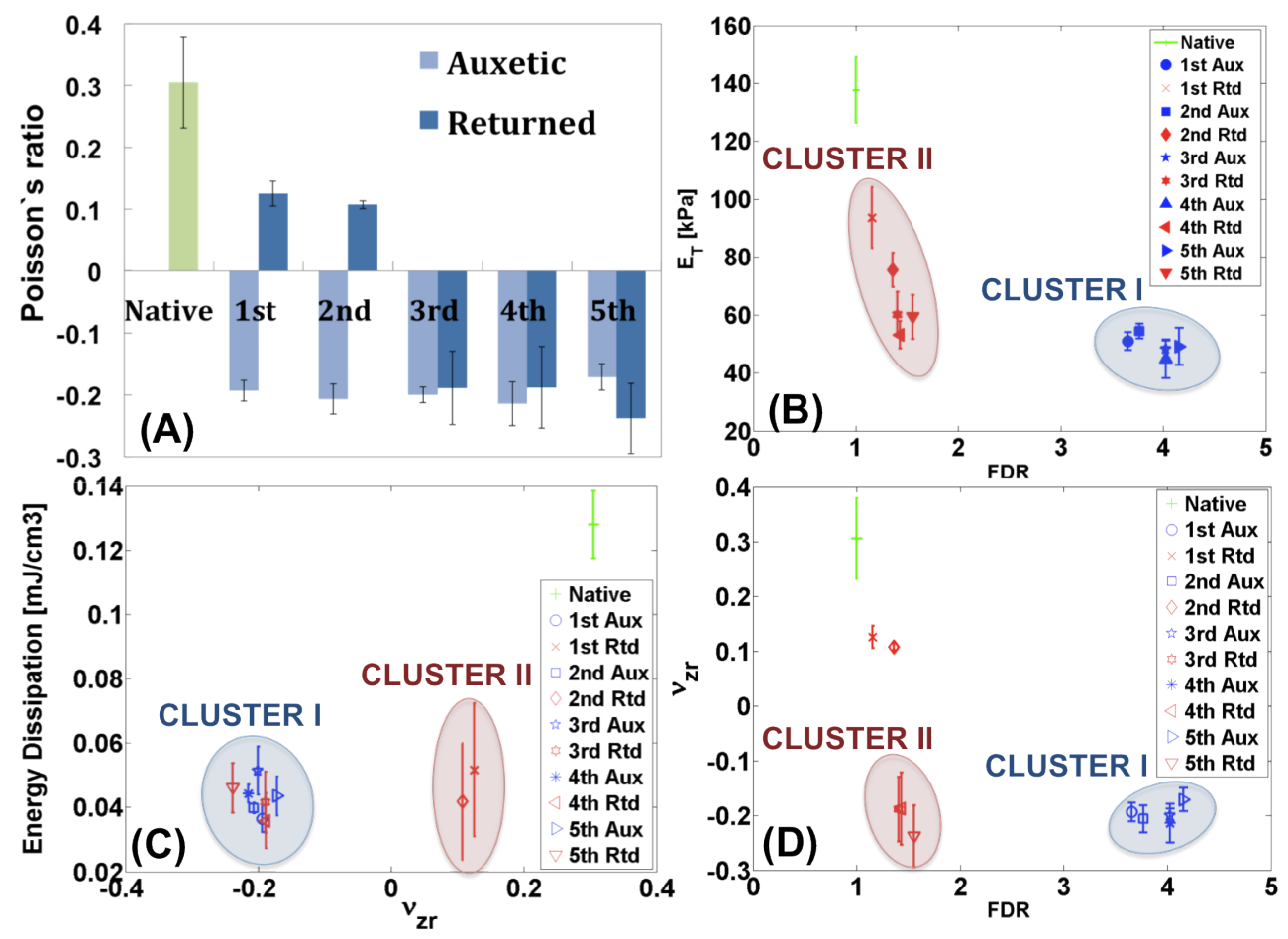

Figure 2: (a) Poisson's ratio of the conventional, auxetic and returned foams; (b) map of the tangent modulus versus the FDR (final density ratio) showing the presence of two distinct clusters of results, the auxetic foams and the returned ones; (c) map describing the distribution of the energy dissipated per unit volume and the Poisson's ratio; (d) the Poisson's ratio versus the FDR. Note that the last three batches of returned foams show a negative Poisson's ratio behaviour rather than a positive one 
compared to the pristine foam case are observed in both the auxetic and returned samples from the $3^{\text {rd }}$ cycle onwards (Figure 2). The SM treatment has also a crucial influence on the mechanical properties, causing a substantial loss in modulus at the first transformation stage (Figure 2). The pristine foam exhibits a modulus of $138 \mathrm{kPa}$, dropping to $51 \mathrm{kPa}$ when transformed into auxetics the first time, and averaging at $49 \mathrm{kPa}$ for the following NPR configurations. The returned foams show also a significant decrease in stiffness compared to the pristine phase (from $94 \mathrm{kPa}$ for the 1st returned and reaching $59 \mathrm{kPa}$ for the 5 th stage). A two-tailed T-test reveals that the differences in stiffness between the $1^{\text {st }}$ and $2^{\text {nd }}$ returned, and between the $3^{\text {rd }}, 4^{\text {th }}$ and $5^{\text {th }}$ returned samples are statistically relevant. The differences in modulus between the auxetic and returned states are however not statistically significant for the $4^{\text {th }}$ and $5^{\text {th }}$ auxetic, and for all the returned phases. The energy dissipated per unit volume during the cyclic loading has the highest value for the pristine foam $\left(0.13 \mathrm{~mJ} \mathrm{~cm}^{-3}\right)$. The $1^{\text {st }}$ NPR shows a significant decrease of the damping capacity by a factor of 3, while the other auxetic phases tend to stabilize around $0.04 \mathrm{~mJ} \mathrm{~cm}^{-3}$. The returned foams have damping capacity steadily decreasing from $0.052 \mathrm{~mJ} \mathrm{~cm}^{-3}$ for the $1 \mathrm{st}$ returned, to an average of $0.042 \mathrm{~mJ} \mathrm{~cm}^{-3}$ for the $4^{\text {th }}$ and $5^{\text {th }}$ phases.

One of the most remarkable aspects is the variation of the Poisson's ratio for the different sets of samples. The Poisson's ratio values related to the foams belonging to the $1^{\text {st }}$ and $2^{\text {nd }}$ SM cycles have $v_{z r}$ averaging around -0.19 (similarly to ${ }^{22}$ ). However, starting from the $3^{\text {rd }}$ reconversion stage, the samples exhibit a negative Poisson's ratio value close to the one of the auxetic samples, while the $5^{\text {th }}$ returned phase has a even lower Poisson's ratio $(-0.24)$, showing that only 3 conversion processes are sufficient to program the auxetic behavior in conventional foams in the returned state. The correlation between the final density ratio $(F D R)$, tangent modulus and Poisson's ratio for all the foams is presented again in Figure 2, in which we observe the presence of two main clusters of data. The first cluster is grouped around a FDR range between 1 and 1.5, and is comprised of the returned samples. Those specimens have a density similar to that of the conventional foam phase. The second cluster collects the auxetic samples, distributed around FDR values ranging between 3.65 and 4.15. The tangent modulus of the 
returned foams decreases for increasing FDR values, whereas the NPR foams show a more constant behavior. While the tangent modulus vs. FDR behaviour of all the foams tends to cluster into two groups, a direct comparison between FDR and Poisson's ratio shows a dependency between the manufacturing parameters used and $v_{z r}$. The $1^{\text {st }}$ and $2^{\text {nd }}$ returned phases represent the first cluster, whereas the second group is comprised of the $3^{\text {rd }}, 4^{\text {th }}$ and $5^{\text {th }}$ returned foams. These last returned phases have the same (negative) Poisson's ratio values as do the foams belonging to the auxetic phase. Significant difference is observed between the energy dissipated per unit volume of the pristine foam and all the subsequent cellular structures. We observe also in this case a clear correlation between energy dissipated and Poisson's ratio of the foams, with the presence of two groups of results, the first one belonging to all the auxetic foams and the $3^{\text {rd }}, 4^{\text {th }}$ and $5^{\text {th }}$ returned, and the second one related to all the positive Poisson's ratio foams. The foams belonging to the first cluster (above) have similar values of energy dissipation. For all these specimens, the Poisson's ratio ranges between -0.24 and -0.17 . The second cluster includes specimens with positive Poisson's ratio (all with $v_{z r}>0.1$ ) and shows values of energy dissipated similar to the ones belonging to the first cluster, but with a significantly higher scatter. The compressive uniaxial behaviour of the auxetic foams shows a bi-linear behavior, missing the central plateau typical of the conventional foams (Figure 3$)^{24}$. While the auxetic foams follow a quasi-bilinear behavior with rapid densification ${ }^{29}$, the returned foams have a stress-strain behavior showing similarities to the one of the pristine foam, especially at strains below $30 \%$. The stiffness levels for these returned foams are however significantly lower ( 5 times on average) than the ones of the auxetic foams at compressive strains higher than $50 \%$.

The SM cycles applied to the PU foams show a clear stabilization of the auxetic behavior for all the specimens after the $3^{\text {rd }} \mathrm{SM}$ treatment. To understand the reasons behind this remarkable behaviour, an elemental $\mathrm{CHN}$ analysis was carried out on the native, $1^{\text {st }}$ and $5^{\text {th }}$ returned samples to investigate for any possible change in the elemental composition that might have occurred after multiple SM cycles. The pristine foam has an average of $66.2 \%$ carbon composition, $8 \%$ hydrogen and $7.1 \%$ nitrogen. After the $1^{\text {st }}$ and $5^{\text {th }}$ SM cycles, the carbon percentage only slightly 

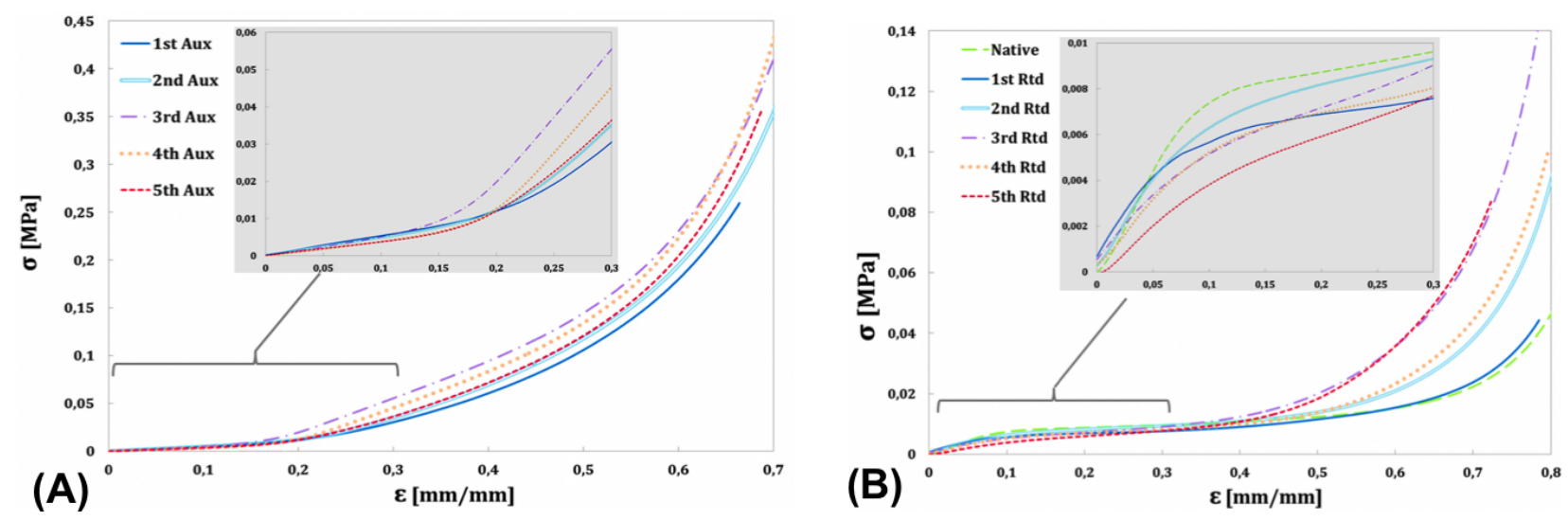

Figure 3: (a) Stress-strain compressive behaviour of (a) auxetic and (b) native and returned SM foams. The auxetic specimens obtained from the $3^{\text {rd }}, 4^{\text {th }}$ and $5^{\text {th }} \mathrm{SM}$ process show a slightly higher stiffness compared to the 1st and 2nd auxetic phases, because of the increase in density. The returned foams (samples belonging to the 1st and 2nd phases) feature the classical linear elastic-plateau-densification compressive behaviour typical of the conventional foam, although a significant hardening (more than $80 \%$ compared to the pristine phase) is present in the $2^{\text {nd }}$ and $3^{\text {rd }}$ returned foams above $60 \%$ of compressive strain. The compressive behaviour of the $4^{\text {th }}$ and $5^{\text {th }}$ returned specimens is 3.7 times higher than the one of the conventional foam for the same $60 \%$ of strain.

increases to $66.6 \%$, with hydrogen and nitrogen content remaining the same, thereby indicating no significant chemical change (degradation) within the composition of the foams. The TGA analysis performed also showed no significant mass loss on heating above $200{ }^{\circ} \mathrm{C}$, and the same result was observed for a conventional foam sample. NPR could be in principle also generated when ribs in the original cell tessellation are missing ("missing-rib model"30), providing a cross chiral-type unit cell that couples uniaxial deformations to rotations and creates the auxetic effect. To examine the possible presence of missing foam ribs due to the SM treatments, 3 samples each belonging to pristine and $5^{\text {th }}$ returned phase batches were placed in a glass container with acetone ${ }^{20}$ for 24 hours. After this treatment, the pristine foam shows a mass loss of $93.3 \%$, while the $5^{\text {th }}$ had $0.21 \%$ only of difference in mass loss, indicating that any possible existing missing rib effect is negligible. Multiphysics properties of polyurethanes are largely affected by the aggregation state of the polymer chains, depending on the chemical specificity of the isocyanate, macroglycol and chain extender units $^{31}$. The microdomain structure of polyurethanes is also highly dependent on the thermal history of the polymer ${ }^{31}$, and the endothermic response of polyurethanes depends on the 
procedure for sample preparation and the details of subsequent conditioning ${ }^{32}$. Melt crystallization may generate different molecular organizations simply by using different thermal cycles $^{33}$. Polymorphic crystal transitions have also been observed in MDI/BD-based polyurethanes, with the initial crystallization tending to form contracted crystal structures, while annealing and stretching the sample favors the formation of extended crystal polymorphs with lower melting point an a structure consistent with the one of BD residues ${ }^{34}$. We have investigated possible differences within the thermal transition temperatures with the DSC on 5 samples from each batch (Figure 4). No significant differences are noticeable in the transition temperatures, leading to the conclusion that no polymorphic changes are occurring within the composition of the ribs, which might have been thought responsible to the SM effect observed. 

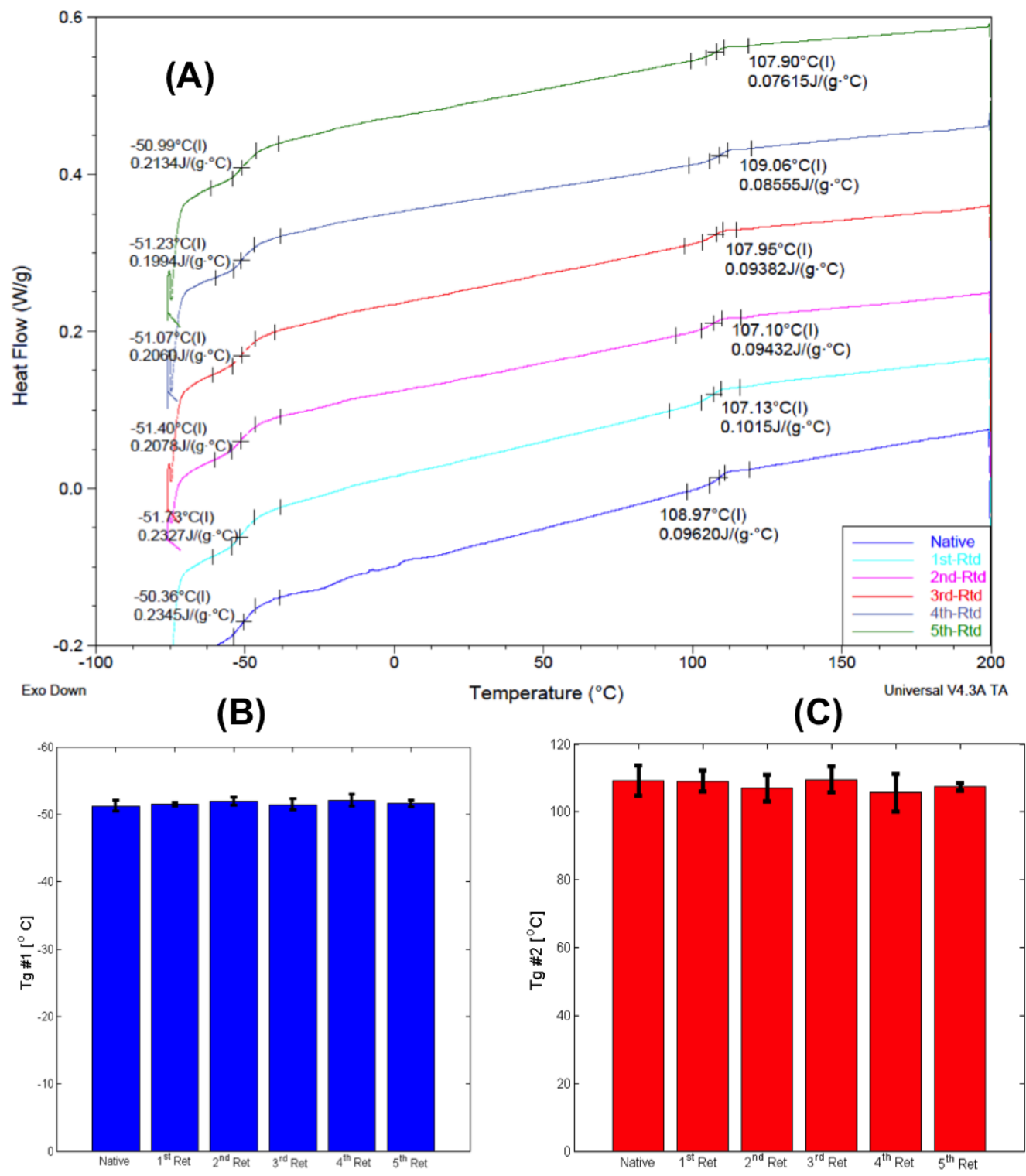

Figure 4: (a) DSC diagram of native and the returned samples under heating. Two second-order transitions are observed, typical of the two-phase structure of the thermoplastic polyurethane, with separated soft and hard phases. The first transition corresponds to a glass transition $\left(\mathrm{T}_{\mathrm{g}}\right)$ of long chains in the soft phase (polyethers and polyols) and appears between $-50{ }^{\circ} \mathrm{C}$ to $-51{ }^{\circ} \mathrm{C}$. At temperatures around $107{ }^{\circ} \mathrm{C}-109{ }^{\circ} \mathrm{C}$, hard crystalline domains containing the urethane groups begin melting for all the foam phases. Negligible changes for the first (b) and second (c) temperatures transitions are observed between the native and the returned foams.

Both small and wide-angle X-ray scattering tests did not show significant changes in cristallinity or the presence of larger scale structures between the various conventional (reference), auxetic and returned foams (Figure 5). The SAXS tests show for all foams the presence of a shoulder, which is 
an indicator of the presence of layers of segregated hard sections alternating the soft section ${ }^{23,35}$. With the Lorentz polarization factor it was possible to observe a peak at $0.08 \AA^{-1}$ common to all the foams considered in this work, which suggests the presence of a phase separated structure with weak interconnections between hard segments as noted in other PU-type foams ${ }^{23}$. The WAXS peaks (Figure 5b) correspond to $4.3 \AA$ and $5.9 \AA$. The value of $4.3 \AA$ is typical of hydrocarbon chain packing, while $5.9 \AA$ is likely related to the bulkiest groups in the hard section. Quite notably and in line with what observed by Li and Zeng for the case of conventional, auxetic and returned $\left(1^{\text {st }}\right)$ foams ${ }^{23}$, all the different porous materials have similar spectra that suggest no intrinsic structural change does occur at the nanoscale and composition level.

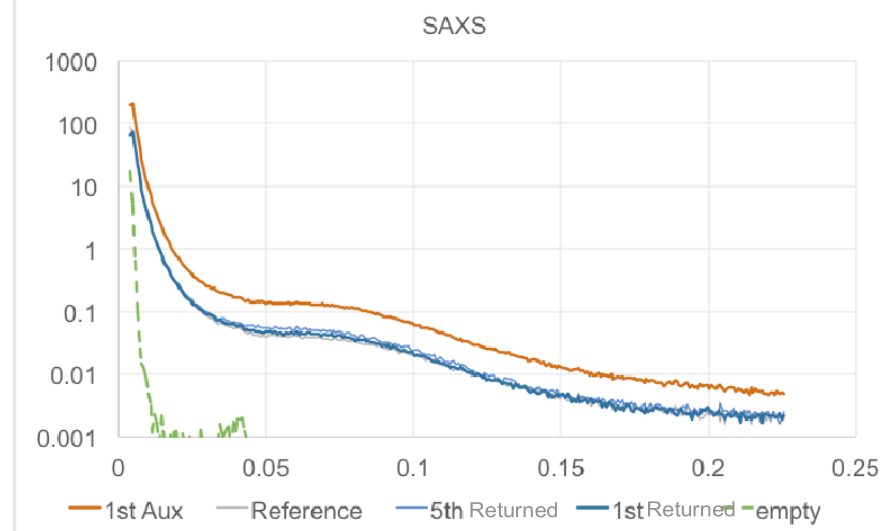

(a)

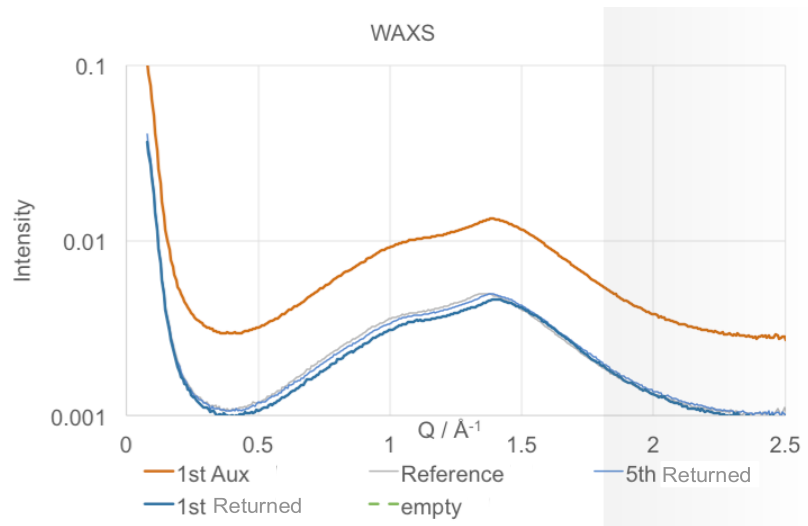

(b)

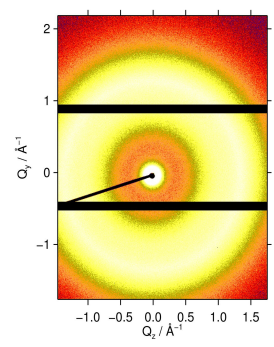

(c)

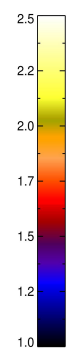

(d)
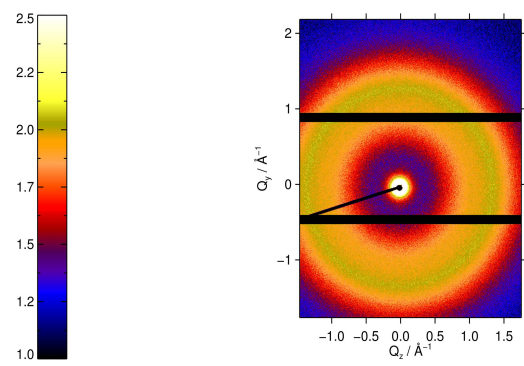

(e)

Figure 5: Logarithmic scales of the (a) SAXS scattered profiles and (b) WAXS patterns intensities for the reference foam, $1^{\text {st }}$ auxetics, $1^{\text {st }}$ returned, $5^{\text {th }}$ returned and baseline instrument configuration. Examples of the WAXS scattered profiles are shown in (c), (d) and (e) for the $1^{\text {st }}$ auxetic, reference and $5^{\text {th }}$ returned respectively. 
A parallel study was performed on the vibrational spectra of the foams in the different auxetic states (Figure 6) and whilst the changes are subtle, there is some evidence for the differences in the microstructure reflected in the data, which are broadly attributed to reduced or increased intramolecular association, particularly through hydrogen bonding. This is shown clearly when considering the superimposed Fourier transform infrared (FT-IR) absorption spectra as the native state. Whilst the spectra do show some variation in baseline position towards the lower frequencies, the good agreement of the baseline above the fingerprint region facilitates direct comparison of the high frequency vibrations. Thus, a consideration of the weak but broad band at 3500-3700 $\mathrm{cm}^{-1}$ (attributed to the hydrogen bonding in the N-H moiety) shows a modest reduction as the native or conventional state makes the transition to the first auxetic state (broken blue line) (Fig. 6a). This is accompanied by an apparent reduction in the intensity and shape of the broad band at $3100-3400 \mathrm{~cm}^{-1}$. The latter is likely to be due to a combination of 'polymeric' O$\mathrm{H}$ and $\mathrm{N}-\mathrm{H}$ bands. Verification was sought through examination of the intensity of the corresponding C-O and C-N stretches (at 1125-1205 $\mathrm{cm}^{-1}$ and $1400 \mathrm{~cm}^{-1}$ respectively) and while there is some corroboration, the variation in baseline position means that this only a qualitative assessment in this region. The carbonyl stretching region (ca. $1700 \mathrm{~cm}^{-1}$ ) is particularly sensitive to changes in electronic environment and the reduction in the hydrogen bonding band is accompanied by a drop in the intensity of the $\mathrm{C}=\mathrm{O}$ stretching band from the conventional to the first auxetic material. There appear to be significant changes in intensity associated with the C-O stretch (1000-1200 $\left.\mathrm{cm}^{-1}\right)$. There are many other, more subtle spectral changes e.g. the profile of the saturated C-H stretching region (immediately below $3000 \mathrm{~cm}^{-1}$ ) also undergoes changes indicating a change in the methyl: methylene: methane ratio. The spectral data support a significant change from the native state to the first auxetic, particularly from a consideration of the $\mathrm{O}-\mathrm{H} / \mathrm{C}-\mathrm{O}$ vibrations, but this is restored in the transition from the first auxetic to the first returned state (Fig. 6b). This suggests that there is a degree of hysteresis present as the apparent relaxation of the polymer network not only restores (but subsequently enhances progressively) the hydrogen bonding response as the network is cycled through subsequent restored states (Fig. 6b). This appears to support the 
aforementioned comments made about the microstructure of the foam in the returned phases (i.e. the change from reticulated topology (rounded cells and membranes) to increasing deformation and wrinkling. The change in surface area, associated packing and potential for hydrogen bonding increases as the structures are cycled. With the $3^{\text {rd }}$ and $4^{\text {th }}$ auxetic there appears to be increases in the vibrational bands at $2962 \mathrm{~cm}^{-1}$ (methyl, C-H stretch) and $1700 \mathrm{~cm}^{-1}$ (carbonyl, $\mathrm{C}=\mathrm{O}$ ).

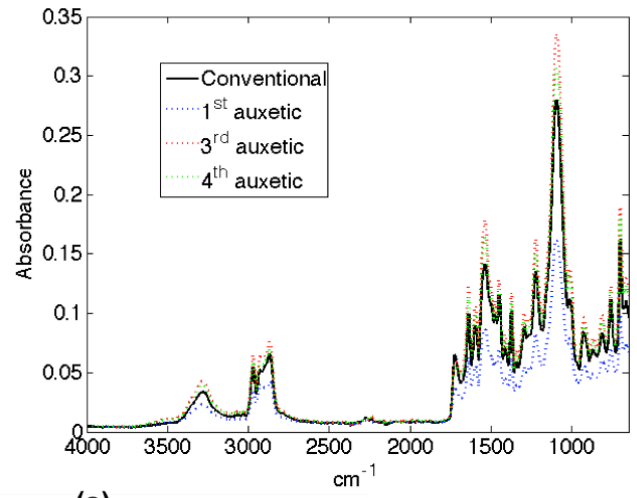

(a)

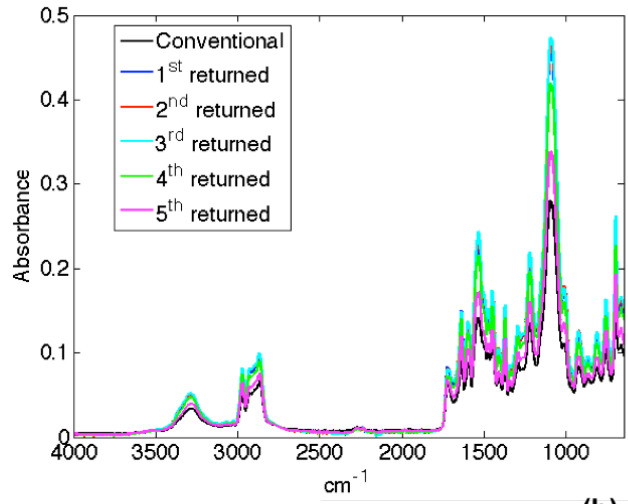

(b)
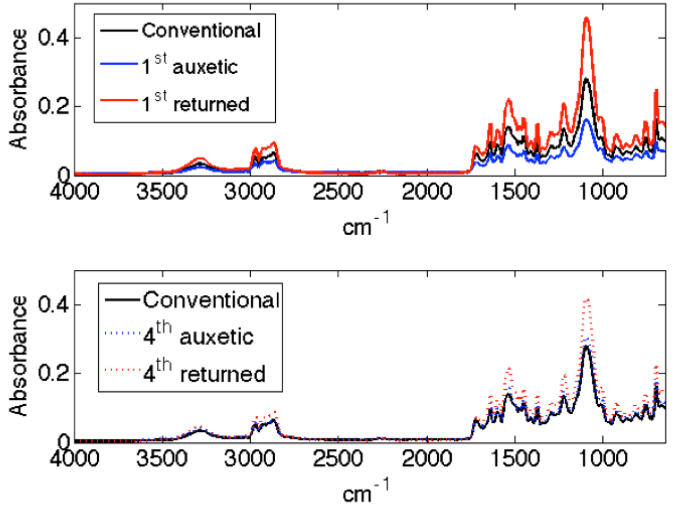

(c)

Figure 6: Absorbance analysis for conventional versus (a) auxetic and (b) returned samples. (c) Direct comparison between the spectra from the conventional, $1^{\text {th }}$ returned and auxetic, and 4 returned and negative Poisson's ratio foams

After the above-mentioned evidence, it is apparent that the unusual blocked shape memory effect has a mechanical origin. At least for points of localized high strains during the cyclic compressions, strain-induced crystallization that is not eliminated on reheating leads after successive cycles to a turnover in auxetic response. To verify any possible topological change in the foam microstructure due this residual crystallization at high strained locations we have carried out CT scans of the native and $5^{\text {th }}$ returned samples (Figure 7). The CT scans highlight more clearly the significant change in the microstructure of the returned foam cells previously observed from the SEM. The native foam 
has more regular cells with Kelvin-type lattice configurations. The structure of the $5^{\text {th }}$ returned foam has slightly elongated concave cells, higher density (pore dimensions are decreased $\begin{array}{llll}\text { between } & 4 \% & \text { and }\end{array}$ 
$\%$ on average) and, quite significantly, the ribs show an irregular cross section distribution and kinks/corrugations along the rib axes. It has been observed in other types of auxetic foams (i.e., $1^{\text {st }}$ auxetic phase) that there are curved ribs and large connecting areas between beams in these foam networks ${ }^{36}$. Strong elongation and ellipticity of the pores has also been observed in anisotropic auxetic foams structures ${ }^{37}$. In classic centresymmetric honeycomb configurations, the auxetic behaviour is due to the global re-entrant shape - i.e., the internal cell angle is negative, giving the cell the butterfly shape typical of auxetic classical configurations ${ }^{38}$. The presence of kinks close to the intersection of the ribs can however produce a negative Poisson's ratio behavior, even though the unit cell of the foam has a global convex behavior with internal positive angles ${ }^{39}$. Figure $7 \mathrm{c}$ shows a multi-reentrant ${ }^{40}$ honeycomb configuration, where the geometry is defined by the global cell wall aspect ratio $(\alpha=h / l)$, relative thickness $(\beta=t / l)$, and the kink aspect ratio $(\gamma=a / l)$.

The cell is also defined by a global internal cell angle $\theta$ and another angle related to the kink position $\phi$. Assuming only bending deformation occurring within the ribs, the in-plane Poisson's ratio $v_{12}$ can be expressed as $v_{12}=[\sin \theta(\alpha+\sin \theta)+2 \gamma \sin \varphi] /[\cos \theta(\cos \theta+2 \gamma \cos \varphi)]^{39,}{ }^{40}$. The expression of the Poisson's ratio $v_{12}$ has been benchmarked by Finite Element simulations $\operatorname{in}^{39,40,41}$. NPR values can be obtained with small kink aspect ratios $\gamma$ and negative king angles $\phi$, even when the baseline configuration of the cell would lead to a positive Poisson's ratio configuration. The kink arm of length $a \sin \phi$ provides a opposing bending moment to the one given by the main ribs $l \sin \theta$ when the unit cell is loaded along the global direction $x_{1}$, generating under appropriate geometry configurations the auxetic behaviour. The indents present in the ribs belonging to the $4^{\text {th }}$ and $5^{\text {th }}$ returned phases cells can represent discontinuities of the elastic axis of the foam ribs, and may be approximated using the corrugated model shown in Figure 7. Kinks and cross-section reduction are produced during the mechanical compression phase within the mould (as also partially observed $\mathrm{in}^{22}$ ), and this would result in a reduction in the restorative force acting to reset the foam to its original dimensions. This would prevent complete shape recovery and would allow relatively facile compressive deformation to a processinduced intrinsic auxetic structure after the first several cycles. The validity of our assertion that the blocked shape memory effect is caused by a mechanical transformation is further compounded by the shape memory induced behavior produced by mechanical training on a syntactic foam 
produced from a styrene thermoset matrix with embedded glass microspheres by $\mathrm{Xu}$ and $\mathrm{Li}^{42}$. In their work the syntactic foam (with original positive Poisson's ratio behaviour) was programmed using a biaxial loading state only that induced a global re-entrant shape to a central portion of the foam section in their mould/crucible. The type of foam metamaterial shown in our work is thermoplastic and open cell, and actually features a blocked SMP effect even when annealing is applied to the specimen. 

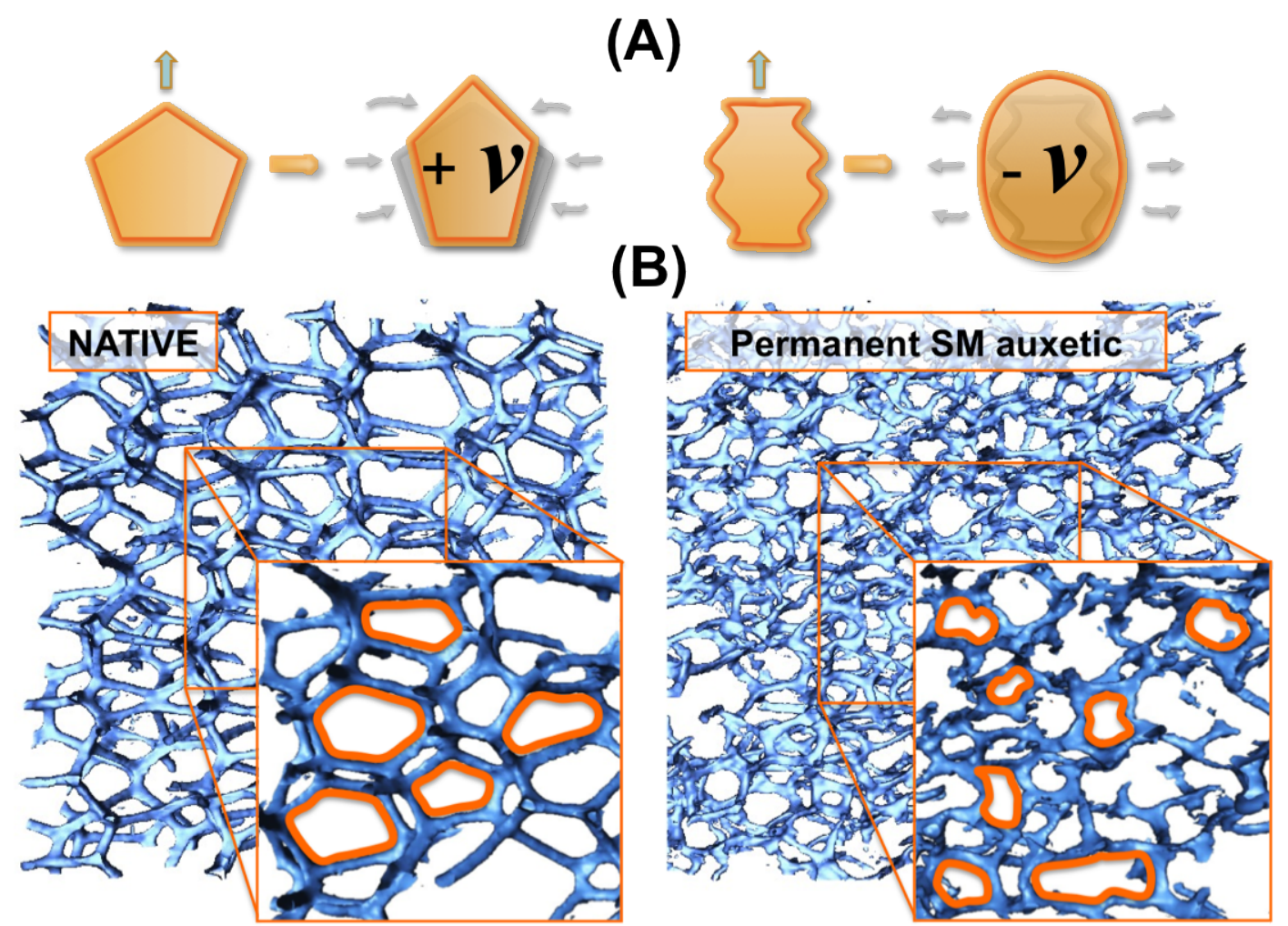

(C)
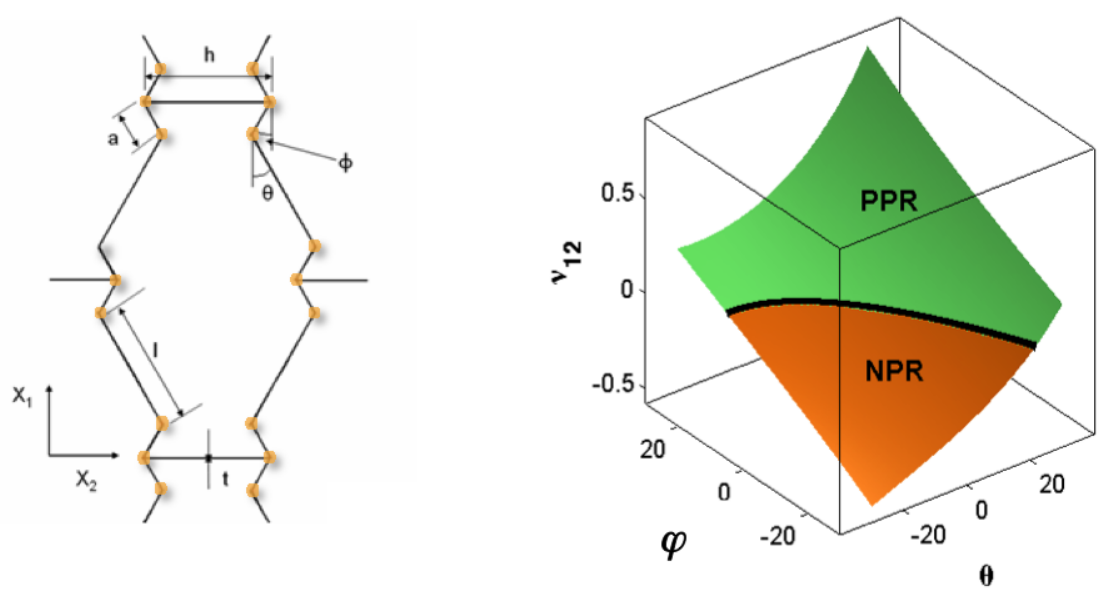

Figure 7: (a) A unit cell of conventional foam exhibiting positive Poisson's ratio $(+v)$. A multiple re-entrant shape leads to an auxetic configuration $(-v)$. (b) Views of $\mu-\mathrm{CT}$ scans of a conventional (native) and returned foam showing for the latter the multi-reentrant shape. (c) Idealised multi-reentrant cell with geometry parameters and the variation of the in-plane Poisson's ratio $v_{12}$ versus the kink angles $\phi$ and internal cell angle $\theta$ (aspect ratio $\alpha=1.0$ and kink aspect ratio $Y=0.5)^{41}$. NPR configurations can be achieved with combinations of positive $\theta$ and $\varphi$ angles. 


\section{Conclusions}

Negative Poisson's ratio open cell PU-PE foam metamaterials with permanent auxetic effect have been produced through multiple shape memory treatments. The permanent NPR effect is justified by the change of the ribs morphology in the returned foam phases with the presence of kinks, indentations and corrugations affecting the shape of the elastic axis of the cell beams. DSC, TGA, SAXS, WAXS, FTIR and elemental analysis do not show any significant change in the polymorphism of the polyurethane. Very negligible mass loss when the samples of the pristine and returned phases were placed in acetone reveals that missing or broken ribs are not responsible for the auxetic behaviour. The new types of auxetic foam metamaterials do show similar negative Poisson's ratio values and energy dissipated per unit volume compared to regular NPR foams. The higher tangent modulus is however a very interesting feature, because auxetic open cell PU foams generally exhibit poor uniaxial stiffness behaviour compared to their pristine conventional phase.

\section{Acknowledgement}

The Ganesha x-ray scattering apparatus used for this research was purchased under EPSRC Grant "Atoms to Applications" Grant ref. EP/K035746/1. The Authors also acknowledge the support of the European Commission FP7 NMP4-LA-2010-246067 M-RECT project for the logistics of the foam manufacturing and mechanical testing, and the UK Royal Society International Collaboration Scheme for the cooperation with Georgia Institute of Technology.

\section{References}

(1) Miyazaki, S.; Fu, Y.; Huang, W. Thin film shape memory alloys: fundamentals and device applications; Cambridge University Press, 2009.

(2) Gil, F. J; Planell, J.A. Shape Memory Alloys for Medical Applications. Proc. Inst. Mech. Eng., Part H 1998, 212(6), 473-488.

(3) L'H oc ine, Y; R y hä ne n, J. In Shape Memory Implants; Yahia, H (Ed), 2000, Springer, New York; Chapter Bioperformance of Shape Memory Alloys, pp.3-23. 
(4) Huang, W. On The Selection of Shape Memory Alloys for Actuators. Mater. Des. 2002, 23(1), $11-19$.

(5) Sun, L.; Huang, W. M.; Ding, Z.; Zhao, Y.; Wang, C. C.; Purnawali, H.; Tang, C. Stimulus Responsive Shape Memory Materials: a Review. Mater. Des. 2012, 33(1), 577-640.

(6) Fan, K.; Huang, W. M.; Wang, C. C.; Al, E. Water-Responsive Shape Memory Hybrid: Design Concept and Demonstration. eXPRESS Polym. Lett. 2011, 5(5), 409-416

(7) Huang, W. M.; Ding, Z.; Wang, C. C.; Wei, J.; Zhao, Y.; Purnawali, H. Shape Memory Materials. Mater. Today 2010, 13(7-8), 54-61.

(8) Wei, Z. G.; Sandstrom, R.; Miyazaki, S. Shape Memory Materials and Hybrid Composites for Smart Systems: Part II Shape-Memory Hybrid Composites. J. Mat. Sci. 1998, 33(15), 3763-3783.

(9) Behl, M.; Lendlein, A. Shape-Memory Polymers. Mater. Today 2007, 10(4), 20-28.

(10) Tey, S.; Huang, W.; Sokolowski, W. Influence of Long-Term Storage in Cold Hibernation on Strain Recovery and Recovery Stress of Polyurethane Shape Memory Polymer Foam. Smart Mater. Struct. 2001, 10, 321.

(11) Huang, W. M. Thermomechanical Behavior of a Polyurethane Shape Memory Polymer Foam. J. Intell. Mater. Syst. Struct. 2006, 17(6-9), 753-760. 
(12) Squeo, E.; Quadrini, F. Solid-State Foaming of Epoxy Resin. J. Cell. Plast. 2008, 44(2), 161.

(13) Tobushi H.; Okumura, K.; Endo, M.; Hayashi, S. Thermomechanical Properties of Polyurethane- Shape Memory Polymer Foam. J. Intell. Mater. Syst. Struct. 2001, 12(4), 283-287.

(14) Li, G.; John, M. A Self-Healing Smart Syntactic Foam Under Multiple Impacts. Compos. Sci. Technol. 2008, 68(15-16), 3337-3343.

(15) Chung, S.; Park, C. The Thermoresponsive Shape Memory Characteristics of Polyurethane Foam. J. Appl. Polym. Sci. 2010, $117(4), 2265-2271$

(16) Lakes, R. S. Foam Structures With a Negative Poisson's ratio. Science 1987, 235, 10381040.

(17) Bianchi, M.; Scarpa, F.; Smith, C.; Whittell, G. Physical and Thermal Effects on the Shape Memory Behaviour of Auxetic Open Cell Foams. J. Mater. Sci. 2010, 45, 341- 347.

(18) Alderson, K.; Alderson, A.; Ravirala, N.; Simkins, V.; Davies, P. Manufacture and Characterisation of Thin Flat and Curved Auxetic Foam Sheets. Phys. Status Solidi (b) 2012, 249(7), $\underline{1315-1321 .}$

(19) Grima, J. N.; Attard, D.; Gatt, R.; Cassar, R. N. A Novel Process for the Manufacture of Auxetic Foams and for their Re-Conversion to Conventional Form. Adv. Eng. Mater. 2009, 11(7), 533-535.

(20) Gatt, R.; Attard, D.; Manicaro, E.; Chetcuti, E.; Grima, J. N. On the Effect of Heat and Solvent Exposure on the Microstructure Properties of Auxetic Foams: A Preliminary Study. 
Phys. Status Solidi (b) 2011, 248(1), 39-44.

(21) Evans, K. E.; Alderson, A. Auxetic Materials: Functional Materials and Structures from Lateral Thinking! Adv. Mater. 2000, 12(9), 617-628.

(22) Bianchi, M.; Scarpa, F.; Smith, C. Shape Memory Behaviour in Auxetic Foams: Mechanical Properties. Acta Mater. 2010, 58(3), $858-865$.

(23) Li, Y.; Zeng, C. On the Successful Fabrication of Auxetic Polyurethane Foams: Materials Requirement, Processing Strategy and Conversion Mechanism Polymer 2016, 87, 98 - 107.

(24) Scarpa, F.; Pastorino, P.; Garelli, A.; Patsias, S.; Ruzzene, M. Auxetic Compliant Flexible PU Foams: Static and Dynamic Properties. Phys. Status Solidi (b) 2005, 242(3), 681-694.

(25) Cadamagnani, F.; Frontoni, S.; Bianchi, M.; Scarpa, F. Compressive Uniaxial Properties of Auxetic Open Cell PU Based Foams. Phys. Status Solidi (b) 2009, 246(9), 2118-2123.

(26) Bianchi, M.; Scarpa, F.; Smith, C. W. Stiffness and Energy Dissipation in Polyurethane Auxetic Foams. J. Mater. Sci. 2008, 43, 5851-5860.

(27) Chan, N.; Evans, K. Fabrication Methods for Auxetic Foams. J. Mater. Sci. 1997, 32, 5945-5953.

(28) McDonald, S.; Ravirala, N.; Withers, P.; Alderson, A. In Situ Three-Dimensional X-ray Microtomography of an Auxetic Foam Under Tension. Scripta Mater. 2009, 60(4), 232 235.

(29) Bezazi, A.; Scarpa, F. Mechanical Behaviour of Conventional and Negative Poisson's Ratio Thermoplastic Polyurethane Foams Under Compressive Cyclic Loading. Int. J. Fatigue 2007, 29(6), $\underline{922-930 .}$ 
(30) Smith, C.; Grima, J.; Evans, K. A Novel Mechanism for Generating Auxetic Behaviour in Reticulated Foams: Missing Rib Foam Model. Acta Mater. 2000, 48(17), 4349 - 4356.

(31) Castonguay, M.; T., K. J.; Zhang, Z.; Laroche, G. In Biomedical Applications of Polyurethanes; Vermette, P., Griesser, H. J., Laroche, G., R., G., Eds.; Volume Tissue Engineering Intelligence Unit 6, Eurekah.com, Georgetown, Texas, Eurekah.com, 2001; Chapter Synthesis, Physicochemical and Surface Characteristics of Polyurethanes, pp 1-20.

(32) Koberstein, J. T.; Galambos, A. F. Multiple Melting in Segmented Polyurethane Block Copolymers. Macromolecules 1992, 25(21), 5618-5624.

(33) Jacques, C. H. M. In Polymer Alloys: Blends, Blocks, Grafts, and Interpenetrating Networks; Klempner, D., Frisch, K. C., Eds.; Plenum Press, New York, 1977; Chapter Effect of Annealing on the Morphology and Properties of Thermoplastic Polyurethanes, p 287.

(34) Blackwell, J.; Lee, C. D. Hard-Segment Polymorphism in MDI/Diol-based Polyurethane Elastomers. J. Polym. Sci., Polym. Phys. Ed. 1984, 22(4), 759-772.

(35) Koberstein, J. T.; Stein, R. S. Small-Angle X-Ray Scattering Studies of Microdomain Structure in Segmented Polyurethane Elastomers. J. Polym. Sci., Polym. Phys. Ed. 1983, 21(8), 1439- 1472.

(36) Grima, J. N.; Gatt, R.; Ravirala, N.; Alderson, A.; Evans, K. Negative Poisson's Ratios in Cellular Foam Materials. Mater. Sci. Eng., A 2006, 423(1-2), 214-218.

(37) Bianchi, M.; Scarpa, F.; Banse, M.; Smith, C. W. Novel Generation of Auxetic Open Cell Foams for Curved and Arbitrary Shapes. Acta Mater. 2011, 59(2), 686-691. 
(38) Masters, I.; Evans, K. Models for the Elastic Deformation of Honeycombs. Compos. Struct. 1996, 35(4), $403-422$.

(39) Bezazi, A.; Scarpa, F.; Remillat, C. A Novel Centresymmetric Honeycomb Composite Structure. Compos. Struct. 2005, 71(3-4), $356-364$.

(40) Innocenti, P.; Scarpa, F. Thermal Conductivity Properties and Heat Transfer Analysis of MultiRe-Entrant Auxetic Honeycomb Structures. J. Compos. Mater. 2009, 43(21), 2419-2439.

(41) Lira, C.; Innocenti, P.; Scarpa, F. Transverse Elastic Shear of Auxetic Multi Re-Entrant Honeycombs. Compos. Struct. 2009, 90(3), 314-322.

(42) Xu, T.; Li, G. A Shape Memory Polymer Based Syntactic Foam with Negative Poisson's ratio. Mater. Sci. Eng. A. 2011, 528, 6804-6811. 


\section{Table of Contents (TOC) graphic}
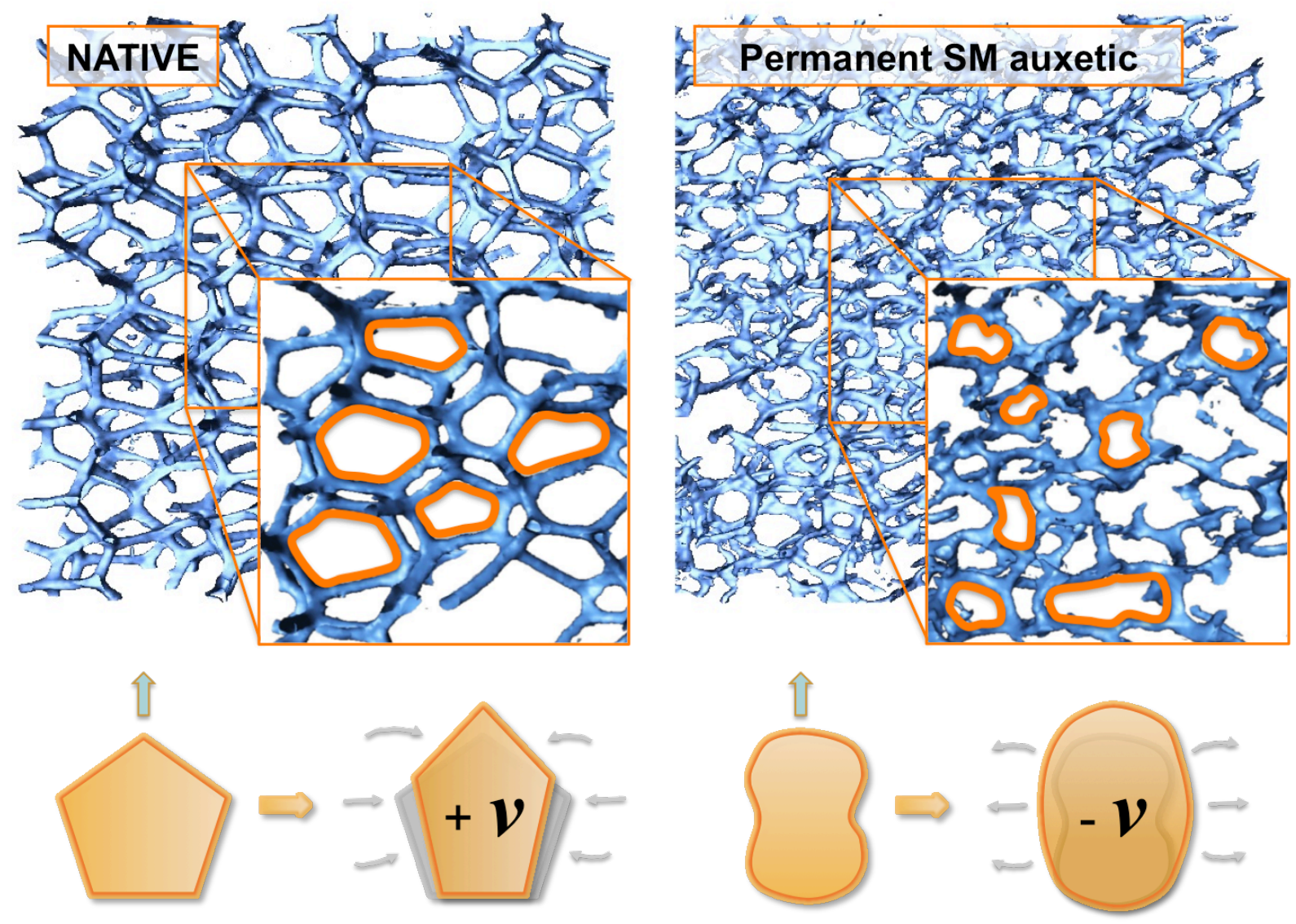